\title{
Phototherapy: Safe and Effective for Challenging Skin Conditions in Older Adults
}

\author{
Sarah W. Matthews, DNP; Kenneth Pike, PhD; Andy J. Chien, MD
}

\section{PRACTICE POINTS}

- With appropriate nursing care, phototherapy can be safe and effective for a variety of conditions in elderly patients.

- Compared to younger patients, elderly patients may need more sessions to achieve comparable clearance rates.

- The increased prevalence of photosensitizing medications in the elderly population will require careful adjustments in dosing.
Identifying safe, effective, and affordable evidence-based dermatologic treatments for older adults can be challenging because of age-related changes. Few studies have examined the effectiveness of phototherapy in older adults. Our retrospective study of patients 65 years and older who were treated with narrowband UVB(NB-UVB) phototherapy aimed to (1) identify the most common dermatologic conditions treated with phototherapy in older adults, (2) examine the effectiveness and safety of phototherapy in older adults, and (3) compare the outcomes to 2 similar studies in the United Kingdom and Turkey.

Cutis. 2021;108:E15-E21.

d

dentifying safe, effective, and affordable evidence-based dermatologic treatments for older adults can be challenging because of age-related changes in the skin, comorbidities, polypharmacy, mobility issues, and cognitive changes. Phototherapy has been shown to be an effective nonpharmacologic treatment option for multiple challenging dermatologic conditions ${ }^{1-8}$; however, few studies have specifically examined its effectiveness in older adults. The challenge for older patients with psoriasis and dermatitis is that the conditions can be difficult to control and often require multiple treatment modalities., ${ }^{9,10}$ Patients with psoriasis also have a higher risk for diabetes, dyslipidemia, and cardiovascular disease compared to other older patients, ${ }^{11,12}$ which poses treatment challenges and makes nonpharmacologic treatments even more appealing.

Recent studies show that phototherapy can help decrease the use of dermatologic medications. Foerster and colleagues ${ }^{2}$ found that adults with psoriasis who were treated with phototherapy significantly decreased their use of topical steroids $(24.5 \%$ fewer patients required steroid creams and $31.1 \%$ fewer patients required psoriasis-specific topicals $)(P<.01)$ while their use of nonpsoriasis-specific medications did not change. Click and colleagues $^{13}$ identified a decrease in medication costs, health care utilization, and risk for immunosuppression in patients treated with phototherapy when compared to those treated with biologics and apremilast. Methotrexate is a common dermatologic medication that is highly associated with increased risks in elderly patients because of impaired immune system function and the presence of comorbidities (eg, kidney disease, obesity, diabetes, fatty liver) ${ }^{14}$ which increase in prevalence with age. Combining phototherapy with methotrexate can substantially decrease the amount of methotrexate needed to achieve disease control, ${ }^{15}$ thereby decreasing the methotrexateassociated risks. Findings from these studies suggest

From the University of Washington, Seattle. Drs. Matthews and Pike are from the School of Nursing. Dr. Chien is from the School of Medicine. Drs. Matthews and Chien also are from Kaiser Permanente Dermatology, Bellevue, Washington.

The authors report no conflict of interest.

Correspondence: Sarah W. Matthews, DNP, University of Washington, 1959 NE Pacific St, Box 357263, Seattle, WA 98195-7263

(sarahm09@uw.edu).

doi:10.12788/cutis.0307 
that a safe, effective, cost-effective, and well-tolerated nonpharmacologic alternative, such as phototherapy, is highly desirable and should be optimized. Unfortunately, most studies that report the effectiveness of phototherapy are in younger populations.

This retrospective study aimed to (1) identify the most common dermatologic conditions treated with phototherapy in older adults, (2) examine the effectiveness and safety of phototherapy in older adults, and (3) compare the outcomes with 2 similar studies in the United Kingdom ${ }^{16}$ and Turkey. ${ }^{17}$

\section{Methods}

Design, Setting, Sample, and Statistical Analysis-The institutional review boards of Kaiser Permanente Washington Health Research Institute, Seattle, and the University of Washington, Seattle, approved this study. It was conducted in a large US multispecialty health care system (Group Health, Seattle, Washington [now Kaiser Permanente Washington]) serving approximately 600,000 patients, using billing records to identify all patients treated with phototherapy between January 1, 2015, and December 31, 2015, all who received narrowband UVB (NB-UVB) phototherapy. All adults 65 years and older who received phototherapy treatment during the 12-month study period were included. Patients were included regardless of comorbidities and other dermatologic treatments to maintain as much uniformity as possible between the present study and 2 prior studies examining phototherapy in older adult populations in the United Kingdom ${ }^{16}$ and Turkey. ${ }^{17}$ Demographic and clinical factors were presented using frequencies (percentages) or means and medians as appropriate. Comparisons of dermatologic conditions and clearance levels used a Fisher exact test. The number of phototherapy treatments to clearance and total number of treatments were compared between groups of patients using independent sample $t$ tests.

Phototherapy Protocol-All patients received treatments administered by specially trained phototherapy nurses using a Daavlin UV Series (The Daavlin Company) or an Ultralite unit (Ultralite Enterprises, Inc), both with 48 lamps. All phototherapy nurses had been previously trained to provide treatments based on standardized protocols (Table 1) and to determine the patient's level of disease clearance using a high to low clearance scale (Table 2). Daavlin's treatment protocols were built into the software that accompanied the units and were developed based on the American Academy of Dermatology guidelines. The starting dose for an individual patient was determined based on the estimated minimal erythema dose for each phototype. If the patient was using photosensitizing medications, then the protocol guided the nurse to start the patient at a lower dose appropriate for their phototype. Patients with vitiligo were treated with the same starting and escalation doses as patients with Fitzpatrick phototype I because of the assumption that their vitiliginous skin had an increased risk for photosensitivity. A more recent review of the evidence has indicated that this assumption was overly conservative, ${ }^{18}$ and Kaiser Permanente Washington's vitiligo protocol has been adjusted.

\section{Results}

Patients-Billing records identified 229 total patients who received phototherapy in 2015, of whom 52 (22.7\%) were at least 65 years old. The median age was 70 years (range, 65-91 years). Twenty-nine (56\%) were men and 35 (67\%) had previously received phototherapy treatments.

Dermatologic Conditions Treated With PhototherapyOur primary aim was to identify the most common dermatologic conditions treated with phototherapy in older adults. Psoriasis and dermatitis were the most common conditions treated in the sample (50\% [26/52] and $21 \%$ [11/52], respectively), with mycosis fungoides being the third most common $(10 \%$ [5/52]) and vitiligo tied with prurigo nodularis as fourth most common (6\% [3/52]) (Figure 1).

Effectiveness and Safety of Phototherapy-Our secondary aim was to examine the effectiveness and safety of phototherapy in older adults. Phototherapy was effective in this population, with 50 of 52 patients ( $96 \%$ ) achieving a high or medium level of clearance. The degree of clearance for each of the dermatologic conditions is shown in Figure 2. Psoriasis and dermatitis achieved high clearance rates in $81 \%(21 / 26)$ and $82 \%(9 / 11)$ of patients, respectively. Overall, conditions did not have significant differences in clearances rates (Fisher exact test, $P=.10$ ). On average, it took patients 33 treatments to achieve medium or high rates of clearance. Psoriasis cleared more quickly, with an average of 30.4 treatments vs 36.1 treatments for other conditions, but the difference was not significant ( $t$ test, $P=.26$ ). Patients received an average of 98 total phototherapy treatments; the median number of treatments was 81 due to many being on maintenance therapy over several months. There was no relationship between a history of treatment with phototherapy and the total number of treatments needed to achieve clearance ( $t$ test, $P=.40$ ), but interestingly, those who had a history of phototherapy took approximately 5 more treatments to achieve clearance. The present study found that a slightly larger number of men were being treated for psoriasis (15 men vs 11 women), but there was no significant difference in response rate based on gender.

Side effects from phototherapy were minimal; 24 patients (46\%) experienced grade 1 (mild) erythema at some point during their treatment course. Thirteen $(25 \%)$ patients experienced grade 2 erythema, but this was a rare event for most patients. Only $1(2 \%)$ patient experienced grade 3 erythema 1 time. Three patients experienced increased itching $(6 \%)$. Thirteen $(25 \%)$ patients had no side effects. None developed severe erythema or blisters, and none discontinued phototherapy because of side effects. Over the course of the study year, we found a high degree of acceptance of phototherapy treatments by 
TABLE 1. Phototherapy Treatment Protocols

\begin{tabular}{|c|c|c|}
\hline Protocol name & Specific steps & Additional details \\
\hline Starting dose & $\begin{array}{l}\text { Fitzpatrick skin phototype determined by referring } \\
\text { provider, then the phototherapy nurse uses a table } \\
\text { that lists estimated MEDs for each skin type and the } \\
\text { starting dose, which is } 70 \% \text { of the MED }\end{array}$ & Phototype I-VI, ${ }^{\text {a }}$ MED not routinely performed \\
\hline Dose escalation & $\begin{array}{l}\text { Subsequent doses were based on the phototype- } \\
\text { driven protocols }\end{array}$ & $\begin{array}{l}\text { Dose increases of } 10 \%-15 \% \text { of the prior dose } \\
\text { based on the nurse's clinical assessment of the } \\
\text { patient at each visit }\end{array}$ \\
\hline $\begin{array}{l}\text { Dose adjustments based } \\
\text { on outcomes }\end{array}$ & $\begin{array}{l}\text { Dose adjuments were based on } 4 \text { variables: } \\
\text { (1) time since the last treatment, (2) patient's } \\
\text { response to the prior doses (same, better, worse), } \\
\text { (3) photosensitizing medications, (4) erythema } \\
\text { and/or itching }\end{array}$ & $\begin{array}{l}\text { Nurse assessed all } 4 \text { variables at each visit and } \\
\text { adjusted the treatments accordingly }\end{array}$ \\
\hline Treatment frequency & $\begin{array}{l}\text { Treatments were administered } 3 \text { times weekly during } \\
\text { the clearance phase }\end{array}$ & Tapered to once weekly once condition controlled \\
\hline \multirow[t]{2}{*}{ Erythema protocol } & If mild erythema occurred but lasted $<24 \mathrm{~h}$ & $\begin{array}{l}\text { Same dose was repeated, then subsequent } \\
\text { doses continued at the prior rate of increase }\end{array}$ \\
\hline & If moderate erythema & $\begin{array}{l}\text { Treatments stopped until symptoms resolved; } \\
\text { once restarted, the dose was decreased to the } \\
\text { prior dose that did not cause erythema, and then } \\
\text { increases were resumed at lower increments; } \\
\text { if previously } 10 \% \text { increases, changed to } 5 \% \text {; if } \\
\text { previously } 15 \% \text { increases, changed to } 10 \%\end{array}$ \\
\hline \multicolumn{3}{|c|}{ Abbreviation: MED, minimal erythema dose. } \\
\hline \multicolumn{3}{|c|}{$\begin{array}{l}\text { aPhototype I: always burns, never tans (vitiligo included in this category), estimated MED of } 175 \mathrm{~mJ} / \mathrm{cm}^{2}\left(\text { starting dose, } 123 \mathrm{~mJ} / \mathrm{cm}^{2}\right) \text {; } \\
\left.\text { II: usually burns, sometimes tans but fades quickly, estimated MED of } 250 \mathrm{~mJ} / \mathrm{cm}^{2} \text { (starting dose, } 175 \mathrm{~mJ} / \mathrm{cm}^{2}\right) \text {; III: sometimes burns, } \\
\left.\text { usually tans and persists longer, estimated MED of } 325 \mathrm{~mJ} / \mathrm{cm}^{2} \text { (starting dose, } 228 \mathrm{~mJ} / \mathrm{cm}^{2}\right) ; \text { IV: moderately pigmented, minimally burns, } \\
\left.\text { tans easily, estimated MED of } 400 \mathrm{~mJ} / \mathrm{cm}^{2} \text { (starting dose, } 122.5 \mathrm{~mJ} / \mathrm{cm}^{2}\right) ; \mathrm{V} \text { : dark brown to black, very darkly pigmented, rarely burns, } \\
\text { estimated MED of } 475 \mathrm{~mJ} / \mathrm{cm}^{2} \text { (starting dose, } 280 \mathrm{~mJ} / \mathrm{cm}^{2} \text { ); VI: darker black, extremely rare burns, estimated MED of } 550 \mathrm{~mJ} / \mathrm{cm}^{2} \\
\text { (starting dose, } 385 \mathrm{~mJ} / \mathrm{cm}^{2} \text { ). }\end{array}$} \\
\hline
\end{tabular}

\section{TABLE 2. High-Low Clearance Scale}

\begin{tabular}{ll}
\hline Level of clearance & Definition \\
\hline High (clear/near) & $\begin{array}{l}>90 \% \text { improvement, or } \\
\text { dermatologic disease } \\
\text { covering }<2 \% \text { BSA }\end{array}$ \\
\hline Medium & $\begin{array}{l}>50 \% \text { improvement } \\
\text { from baseline }\end{array}$ \\
\hline Low & $\begin{array}{l}\text { Persistent scaly, thick, red, } \\
\text { or itchy lesions in the case } \\
\text { of psoriasis, dermatitis, } \\
\text { mycosis fungoides, or } \\
\text { prurigo nodularis; persistent } \\
\text { depigmented macules and } \\
\text { patches in the case of vitiligo }\end{array}$ \\
\hline
\end{tabular}

Abbreviation: BSA, body surface area.

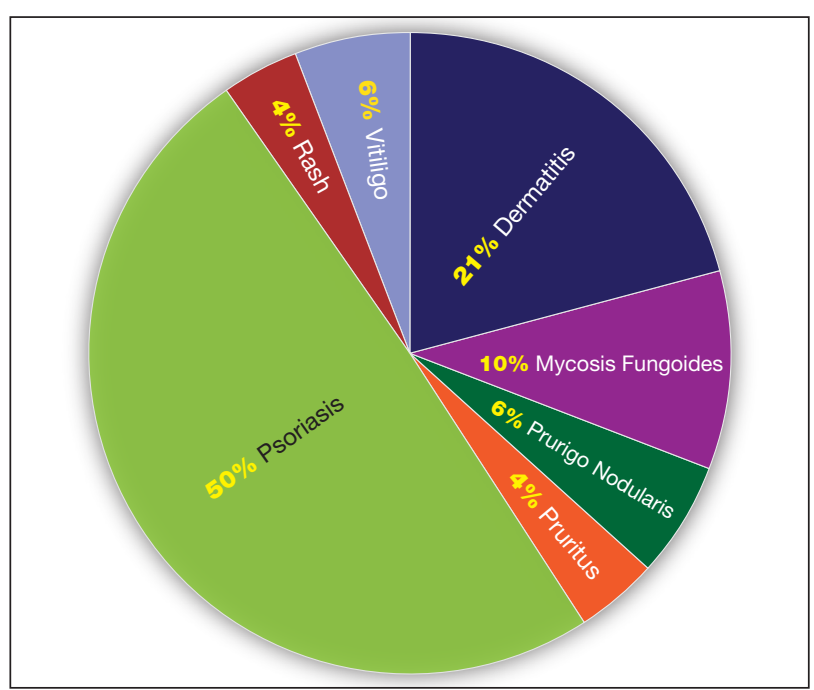

FIGURE 1. Dermatologic conditions of older patients $(N=52)$. Percentages were rounded to the nearest whole number. 


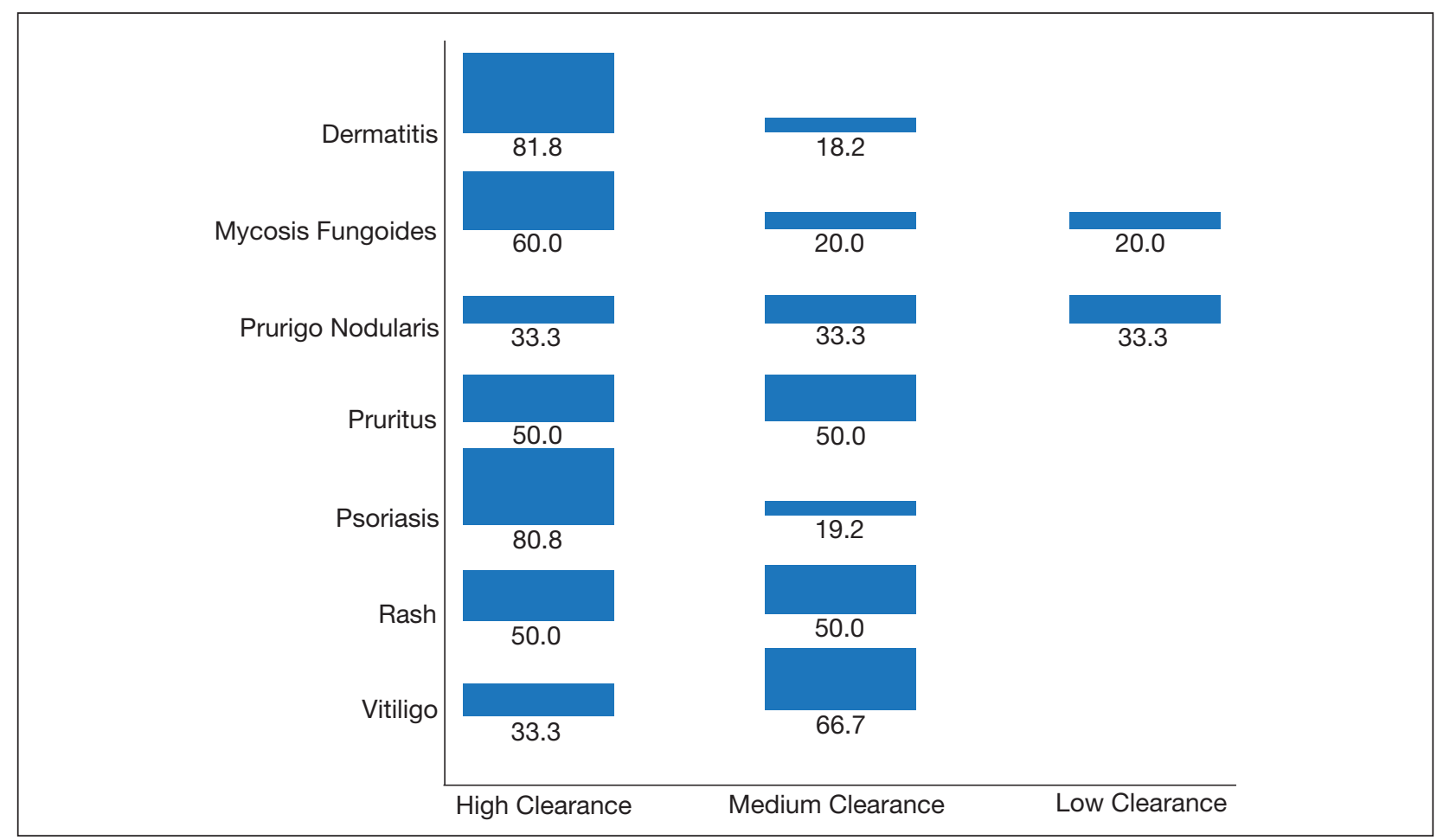

FIGURE 2. Degree of clearance by dermatologic condition.

TABLE 3. Comparison of Conditions Treated

\begin{tabular}{|c|c|c|c|}
\hline Condition treated & $\begin{array}{l}\text { Current study } \\
(\mathrm{N}=52),{ }^{\text {a }} \mathrm{n}(\%)\end{array}$ & $\begin{array}{l}\text { United Kingdom study }{ }^{16} \\
(\mathrm{~N}=37),{ }^{\mathrm{b}} \mathrm{n}(\%)\end{array}$ & $\begin{array}{l}\text { Turkey study }{ }^{17} \\
(\mathrm{~N}=95),{ }^{\mathrm{b}} \mathrm{n}(\%)\end{array}$ \\
\hline Psoriasis & $26(50)$ & $19(51)$ & 37 (39) \\
\hline Dermatitis & (21) & $4(11)$ & $6(6)$ \\
\hline MF & $5(10)^{c}$ & NA & $29(30)$ \\
\hline PN & $3(6)^{c}$ & $4(11)$ & NA \\
\hline Vitiligo & $3(6)^{c}$ & NA & NA \\
\hline Pruritus & $2(4)^{c}$ & $4(11)$ & $12(13)$ \\
\hline Nonspecific rash & $2(4)^{c}$ & NA & NA \\
\hline Grover disease & NA & $2(5)$ & NA \\
\hline $\begin{array}{l}\text { Other (scleromyxedema, polymorphic light } \\
\text { eruption, granuloma annulare, erythroderma) }\end{array}$ & NA & $4(11)$ & NA \\
\hline Lichen planus & NA & NA & $8(8)$ \\
\hline $\begin{array}{l}\text { Other (morphea, keratoderma, pigmented } \\
\text { purpuric dermatosis) }\end{array}$ & NA & NA & $3(3)$ \\
\hline $\begin{array}{l}\text { Abbreviations: NB-UVB, narrowband UVB; PUVA } \\
\text { 'NB-UVB phototherapy only. } \\
\text { 'C Combination of NB-UVB and PUVA. } \\
\text { 'Percentages rounded to nearest whole number. }\end{array}$ & , psoralen plus UV & osis fungoides; NA, not availa & PN, prurigo nodula \\
\hline
\end{tabular}


older patients: 22 (42\%) completed therapy after achieving clearance, $10(19 \%)$ were continuing ongoing treatments (maintenance), and 15 (29\%) stopped because of life circumstances (eg, other health issues, moving out of the area). Only 4 (8\%) stopped because of a lack of effectiveness, and $1(2 \%)$ patient because the treatments were burdensome.

Comparison of Outcomes-Our third aim was to compare the outcomes with similar studies in the United Kingdom ${ }^{16}$ and Turkey. ${ }^{17}$ This study confirmed that phototherapy is being used in older adults $(22.7 \%$ of this study's total patients) and is an effective treatment for older patients experiencing a range of challenging inflammatory and proliferative skin diseases similar to studies in the general population. Prior phototherapy studies in elderly patients also found psoriasis to be the most common skin condition treated, with 1 study finding that $51 \%$ $(19 / 37)$ of older phototherapy patients had psoriasis, ${ }^{16}$ while another reported 58\% (37/95) of older phototherapy patients had psoriasis. ${ }^{17}$ These numbers are similar to those in our study, which showed $50 \%$ (26/52) of elderly phototherapy patients had psoriasis. Psoriasis is the main indication for treatment with NB-UVB phototherapy in the general population, ${ }^{19}$ and because the risk for psoriasis increases with age, ${ }^{20}$ it is not surprising that all 3 studies found psoriasis to be the most common indication in elderly phototherapy patients. Table 3 provides further details on conditions treated in all 3 studies.

\section{Comment}

Our study found that $94 \%$ of patients with psoriasis achieved clearance with an average of 30.4 treatments, which is comparable to the reported $91 \%$ response rate with an average of 30 treatments in the United Kingdom. ${ }^{16}$ The other similar study in Turkey ${ }^{17}$ reported $73.7 \%$ of psoriasis patients achieved a $75 \%$ or more improvement from baseline with an average of 42 treatments, which may reflect underlying differences in regional skin type. Of note, the scatter chart (Figure 3) shows that several patients in the present study's analysis are listed as not clear, but many of those patients had low treatment numbers below the mean time to clearance. Thus, the present study's response rate may have been underestimated.

In the general population, studies show that psoriasis treated with standardized phototherapy protocols typically clears with an average of 20.6 treatments. ${ }^{21}$

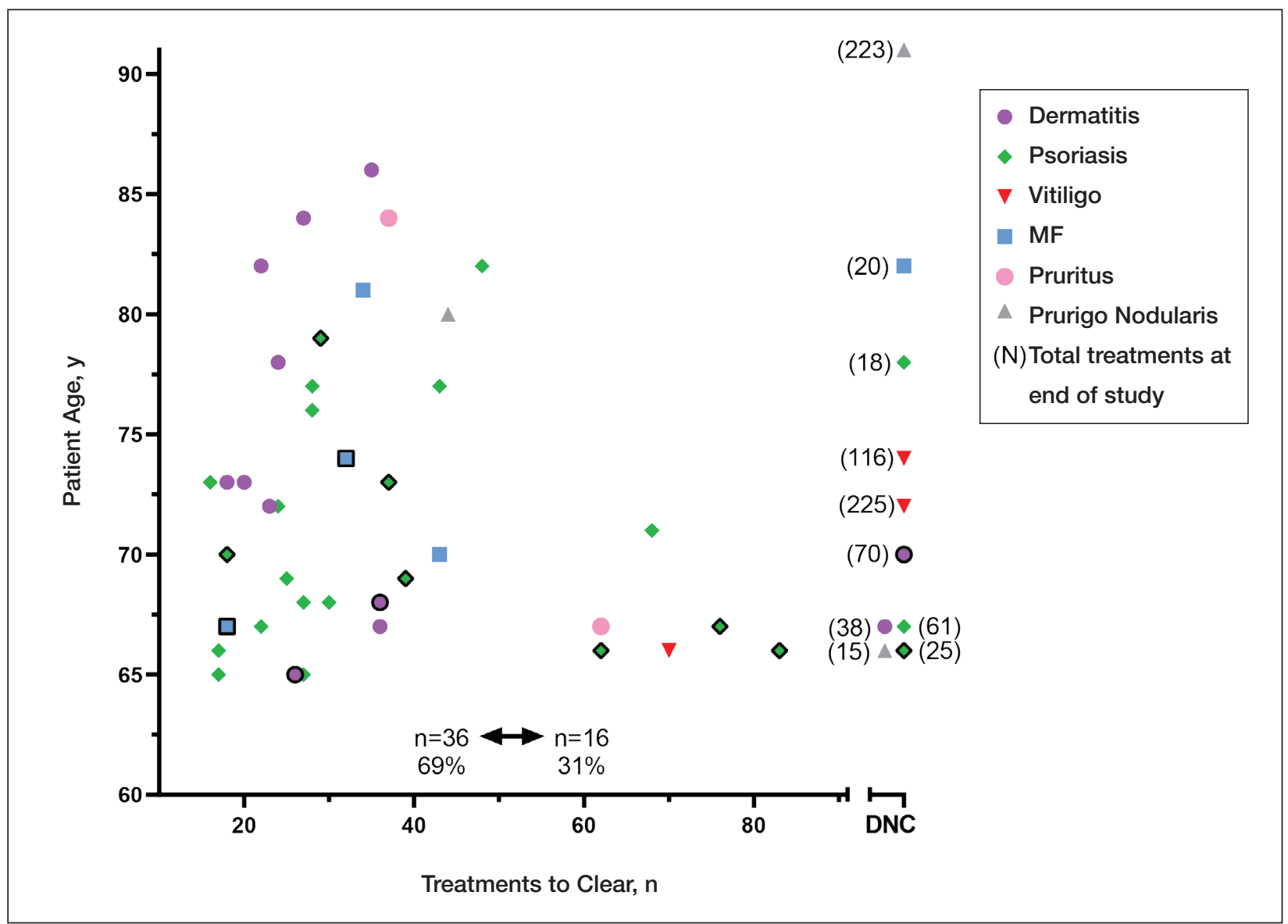

FIGURE 3. Comparison of total treatments and side effects across all conditions. MF indicates mycosis fungoides; DNC, did not clear. Bold rule indicates patients who experienced side effects greater than grade 1. 
The levels of clearance were similar in our study's older population, but more treatments were required to achieve those results, with an average of 10 more treatments needed (an additional 3.3 weeks). Similar results were found in this sample for dermatitis and mycosis fungoides, indicating comparable clearance rates and levels but a need for more treatments to achieve similar results compared to the general population.

Additionally, in the current study more patients experienced grade 1 (mild) erythema (46\%) and grade 2 erythema $(25 \%)$ at some point in their treatment compared with the United Kingdom ${ }^{16}(1.89 \%)$ and Turkey ${ }^{17}$ (35\%) studies, though these side effects did not impact the clearance rate. Interestingly, the current study's scatter chart (Figure 3) illustrates that this side effect did not seem to increase with aging in this population. If anything, the erythema response was more prevalent in the median or younger patients in the sample. Erythema may have been due to the frequent use of photosensitizing medications in older adults in the United States, some of which typically get discontinued in patients 75 years and older (eg, statins). Other potential causes might include the use of phototype vs minimal erythema dose-driven protocols, the standard utilization of protocols originally designed for psoriasis vs other condition-specific protocols, missed treatments leading to increased sensitivity, or possibly shielding mishaps (eg, not wearing a prescribed face shield). Given the number of potential causes and the possibility of overlapping factors, careful analysis is important. With NB-UVB phototherapy, near-erythemogenic doses are optimal to achieve effective treatments, but this delicate balance may be more problematic for older adults. Future studies are needed to fully determine the factors at play for this population. In the interim, it is important for phototherapy-trained nurses to consider this risk carefully in the older population. They must follow the prescribed protocols that guide them to query patients about their responses to the prior treatment (eg, erythema, tenderness, itching), photosensitizing medications, missed treatments, and placement of shielding, and then adjust the treatment dosing accordingly.

Limitations-This study had several limitations. Although clinical outcomes were recorded prospectively, the analysis was retrospective, unblinded, and not placebo controlled. It was conducted in a single organization (Group Health [now Kaiser Permanente Washington]) but did analyze data from 4 medical centers in different cities with diverse demographics and a variety of nursing staff providing the treatments. Although the vitiligo treatment protocol likely slowed the response rate for those patients with vitiligo, the numbers were small (ie, only 3 of 52 patients), so the researchers chose to include them in the current study. The sample population was relatively small, but when these data are evaluated alongside the studies in the United Kingdom ${ }^{16}$ and Turkey, ${ }_{17}$ they show a consistent picture illustrating the effectiveness and safety of phototherapy in the older population. Further epidemiologic studies could be helpful to further describe the usefulness of this modality compared with other treatments for a variety of dermatoses in this age group. Supplementary analysis specifically examining the relationship between the number and type of photosensitizing medications, frequency of erythema, and time to clearance also could be useful.

\section{Conclusion}

Older adults with a variety of dermatoses respond well to phototherapy and should have the opportunity to use it, particularly considering the potential for increased complications and costs from other treatment modalities, such as commonly used immunosuppressive pharmaceuticals. However, the current study, and the comparison studies indicate that it is important to carefully consider the slower clearance rates and the potential risk for increased erythema in this population and adjust patient education and treatment dosing accordingly.

Unfortunately, many dermatology centers do not offer phototherapy because of infrastructure limitations such as space and specially trained nursing staff. Increasing accessibility of phototherapy for older adults through home treatments may be an alternative, given its effectiveness in the general population..$^{22,23}$ In addition, home phototherapy may be worth pursuing for the older population considering the challenges they may face with transportation to the clinic setting and their increased risk for serious illness if exposed to infections such as COVID-19. The COVID-19 pandemic has brought to light the need for reliable, safe, and effective treatments that can be utilized in the safety of patients' homes and should therefore be considered as an option for older adults. Issues such as mobility and cognitive decline could pose some complicating factors, but with the help of a well-trained family member or caregiver, home phototherapy could be a viable option that improves accessibility for older patients. Future research opportunities include further examination of the slower but ultimately equivalent response to phototherapy in the older population, the influence of photosensitizing medications on phototherapy effects, and the impact of phototherapy on utilization of immunosuppressive pharmaceuticals in older adults.

\section{REFERENCES}

1. British Photodermatology Group. An appraisal of narrowband (TL-01) UVB phototherapy. British Photodermatology Group Workshop Report (April 1996). Br J Dermatol. 1997;137:327-330.

2. Foerster J, Boswell K, West J, et al. Narrowband UVB treatment is highly effective and causes a strong reduction in the use of steroid and other creams in psoriasis patients in clinical practice. PLOS ONE. 2017;12:e0181813. doi:10.1371/journal.pone.0181813

3. Fernández-Guarino M, Aboin-Gonzalez S, Barchino L, et al. Treatment of moderate and severe adult chronic atopic dermatitis with narrow-band UVB and the combination of narrow-band UVB/UVA phototherapy. Dermatol Ther. 2015;29:19-23.

4. Ryu HH, Choe YS, Jo S, et al. Remission period in psoriasis after multiple cycles of narrowband ultraviolet B phototherapy. J Dermatol. 2014;41:622-627. 
5. Tintle S, Shemer A, Suárez-Fariñas M, et al. Reversal of atopic dermatitis with narrow-band UVB phototherapy and biomarkers for therapeutic response. J Allergy Clin Immunol. 2011;128:583-593.

6. Gambichler T, Breuckmann F, Boms S, et al. Narrowband UVB phototherapy in skin conditions beyond psoriasis. J Am Acad Dermatol. 2005;52:660-670.

7. Schneider LA, Hinrichs R, Scharffetter-Kochanek K. Phototherapy and photochemotherapy. Clin Dermatol. 2008;26:464-476.

8. Martin JA, Laube S, Edwards C, et al. Rate of acute adverse events for narrow-band UVB and psoralen-UVA phototherapy. Photodermatol Photoimmunol Photomed. 2007;23:68-72.

9. Mokos ZB, Jovic A, Ceovic R, et al. Therapeutic challenges in the mature patient. Clin Dermatol. 2018;36:128-139.

10. Di Lernia V, Goldust M. An overview of the efficacy and safety of systemic treatments for psoriasis in the elderly. Exp Opin Biol Ther. 2018;18:897-903

11. Napolitano M, Balato N, Ayala F, et al. Psoriasis in elderly and nonelderly population: clinical and molecular features. G Ital Dermatol Venereol. 2016;151:587-595.

12. Grozdev IS, Van Voorhees AS, Gottlieb AB, et al. Psoriasis in the elderly: from the Medical Board of the National Psoriasis Foundation. J Am Acad Dermatol. 2011;65:537-545.

13. Click J, Alabaster A, Postlethwaite D, et al. Effect of availability of athome phototherapy on the use of systemic medications for psoriasis. Photodermatol Photoimmunol Photomed. 2017;33:345-346.
14. Piaserico S, Conti A, Lo Console F, et al. Efficacy and safety of systemic treatments for psoriasis in elderly. Acta Derm Venereol. 2014;94:293-297.

15. Soliman A, Nofal E, Nofal A, et al. Combination therapy of methotrexate plus NB-UVB phototherapy is more effective than methotrexate monotherapy in the treatment of chronic plaque psoriasis. J Dermatol Treat. 2015;26:528-534.

16. Powell JB, Gach JE. Phototherapy in the elderly. Clin Exp Dermatol. 2015;40:605-610.

17. Bulur I, Erdogan HK, Aksu AE, et al. The efficacy and safety of phototherapy in geriatric patients: a retrospective study. An Bras Dermatol. 2018;93:33-38

18. Madigan LM, Al-Jamal M, Hamzavi I. Exploring the gaps in the evidence-based application of narrowband UVB for the treatment of vitiligo. Photodermatol Photoimmunol Photomed. 2016;32:66-80.

19. Ibbotson SH. A perspective on the use of NB-UVB phototherapy vs. PUVA photochemotherapy. Front Med (Lausanne). 2018;5:184.

20. Bell LM, Sedlack R, Beard CM, et al. Incidence of psoriasis in Rochester, Minn, 1980-1983. Arch Dermatol. 1991;127:1184-1187.

21. Totonchy MB, Chiu MW. UV-based therapy. Dermatol Clin. 2014;32:399-413.

22. Cameron H, Yule S, Dawe RS, et al. Review of an established UK home phototherapy service 1998-2011: improving access to a cost-effective treatment for chronic skin disease. Public Health. 2014;128:317-324.

23. Matthews SW, Simmer M, Williams L, et al. Transition of patients with psoriasis from office-based phototherapy to nurse-supported home phototherapy: a pilot study. JDNA. 2018;10:29-41. 